\title{
Evolución en el diagnóstico del trastorno afectivo bipolar entre 2010 y 2015. Caldas, Colombia
}

\author{
Evolution in the diagnosis of bipolar affective disorder between 2010 and 2015. Caldas, Colombia
}

José Henry Osorio ${ }^{1,2 *}$ orcid.org/0000-0002-6875-3215

Yoccner Edilson Quenan' orcid.org/0000-0002-8047-0306

Marco Antonio Acosta ${ }^{3}$ orcid.org/0000-0001-5374-3586

1 Laboratorio de Investigación en Metabolismo. Universidad de Manizales. Manizales, Colombia

2 Laboratorio de Investigación Bioquímica Clínica. Universidad de Caldas. Manizales, Colombia

3 Fundación para la atención de la salud mental comportamiento y desarrollo humano - FADESCO

\section{Resumen}

Introducción: En el departamento de Caldas, Colombia, se ha observado un incremento notable en el diagnóstico del trastorno afectivo bipolar (TAB), en los últimos años. Objetivo: Analizar el perfil epidemiológico del TAB en Caldas entre los años 2010 y 2015. Materiales y métodos: Se consultó la base de datos de la Dirección Territorial de Salud de Caldas y los registros del ministerio salud y protección social, para TAB. Se buscó la incidencia y la prevalencia entre los años 2010 y 2015 y se comparó la presentación de acuerdo al tipo de TAB. Resultados: La prevalencia de TAB en el departamento de Caldas el 2010 fue en general de $0,03 \%$ y para mayores de 18 años fue de $0,08 \%$; para el 2015 en la población general, ascendió a 0,10\% y para mayores de 18 años ascendió a 0,22\%. La incidencia estuvo determinada por un incremento de 342 pacientes en 2010 a 969 pacientes en el 2015. Conclusiones: Se observa un marcado incremento del diagnóstico de TAB en Caldas, posiblemente debido a problemas socioculturales, causas epigenéticas o problemas de formulación tipo off-label.

Palabras clave: Salud mental; epidemiología; incidencia; prevalencia; trastorno bipolar. (Fuente: DeCS, Bireme).

\begin{abstract}
Introduction: Caldas, Colombia has seen an increasing number of diagnoses of bipolar disorder. Objective: The present study analyzes the epidemiological profile for bipolar disorder in Caldas between the years 2010 and 2015. Materials and methods: The data base of the Dirección Territorial de Salud de Caldas was consulted for cases of bipolar disorder. The incidence and prevalence for bipolar disorder was established, and a comparison by each subtype of bipolar disorder was performed. Results: The prevalence for bipolar disorder in Caldas was $0.03 \%$ for the general population, and $0.08 \%$ for cases older than 18 years, which reflects an increment of $0.10 \%$ in 2015 for the general population and $0.22 \%$ for those patients older than 18 years. The incidence was determined by an increment in the number of patients, from 342 in 2010 to 969 in 2015. Conclusions: An increasing number of patients between 2010 and 2105 were observed, probably due to sociocultural problems, epigenetic causes or prescription practices such as off label practices.
\end{abstract}

Keywords: Mental health; epidemiology; incidence; prevalence; schizophrenia; bipolar disorder. (Source: DeCS, Bireme).

\footnotetext{
*Autor de correspondencia

José Henry Osorio

e-mail: josorio@umanizales.edu.co
} 


\section{Introducción}

Un episodio de manía es un episodio diferenciado de un estado de ánimo anormal y persistentemente elevado, expansivo o irritable, caracterizado por un aumento exagerado de la autoestima, disminución de la necesidad de sueño, facilidad para distraerse, gran actividad física y mental, e implicación excesiva en comportamientos placenteros, que dura al menos una semana y está presente la mayor parte del día, casi todos los días(1).

Un episodio de hipomanía dura al menos 4 días y es similar al episodio de manía, excepto en que no es suficientemente grave como para causar un deterioro del funcionamiento social o laboral y no hay síntomas psicóticos(2). El trastorno de depresión mayor se caracteriza por al menos 4 síntomas de una lista en la que se incluyen cambios en el apetito y el peso, en el sueño y la actividad, falta de energía, sentimientos de culpa, problemas para pensar y tomar decisiones, y pensamientos recurrentes de muerte o suicidio(3).

Se considera el trastorno afectivo bipolar (TAB), como un trastorno del estado de ánimo caracterizado por la presencia de manía, o hipomanía y episodios alternantes de depresión, es decir que el afectado suele oscilar entre la alegría y la tristeza de una manera mucho más notable que las personas del promedio de la población(4).

El TAB se divide a su vez en TAB I, el cual presenta una evolución clínica, de uno o más episodios de manía con episodios depresivos mayores ocasionalmente, y TAB II que se caracteriza por la presencia de episodios de depresión mayor e hipomanía en lugar de manía(5).

El TAB es una de las enfermedades mentales más comunes, severas y persistentes; el trastorno tiende a ser una condición permanente a lo largo de la vida y comienza típicamente en la adolescencia o en la adultez temprana ${ }^{(6)}$. Es una enfermedad caracterizada por altas tasas disfuncionalidad, ansiedad comórbida, recaídas, trastorno por abuso de sustancias, y mortalidad prematura debida especialmente a la alta tasa de $\operatorname{suicidio}^{(7)}$.

Por otro lado, la incidencia de una enfermedad, es la medida del número de nuevos casos que ocurren en una población durante un periodo de tiempo de observación(8), esto aplicado a la población en general, mientras que la prevalencia determina el número total de personas que sufren la enfermedad, en un momento dado o durante un período de tiempo determinado ${ }^{(9)}$.

La prevalencia es una proporción, y se obtiene dividiendo el número total de individuos enfermos por el total de la población (incluido el número de enfermos). Ciertos factores como la edad de las poblaciones, las tasas de mortalidad, así como los patrones de migración, influyen en el cálculo de la prevalencia; lo que a su vez puede diferir entre los sitios evaluados y variar dentro de las poblaciones donde se determine ${ }^{(10)}$.

Ambas determinaciones son valiosas como en este estudio, donde se analiza la evolución en el diagnóstico del trastorno afectivo bipolar en el departamento de Caldas (Colombia) entre los años 2010 y 2015, toda vez que se ha detectado un incremento en el número de casos de esta patología mental en el departamento. Caldas es uno de los 32 departamentos que, conforman la República de Colombia, su capital es Manizales, y está ubicado en el centro del país, en la región andina, con 7888 km² y pertenece al eje cafetero.

Según Bauer et al.(11), sin discriminación del origen étnico, la nacionalidad o el estatus socio-económico, el $\mathrm{TAB}$ afecta alrededor del $1 \%$ de la población, siendo una de las mayores causas de discapacidad entre la gente joven. La incidencia del suicidio entre pacientes con $\mathrm{TAB}$ es alta, siendo 20 veces mayor que en la población general(12-14).

El trastorno bipolar es uno de los trastornos mentales más comunes, severos y persistentes. La prevalencia se estima entre un 0,3 y un $7 \%$ de la población general(15). Por lo anterior, el presente estudio tiene como objetivo analizar el perfil epidemiológico del TAB en el Departamento de Caldas entre los años 2010 y 2015.

\section{Materiales y métodos}

El presente estudio es de tipo retrospectivo, longitudinal, descriptivo y analítico. Se obtuvo la información por municipios de las IPS (Instituciones Prestadoras de Salud) del Departamento, de la base de datos de la Dirección Territorial de Salud de Caldas (DTSC) y los registros del Ministerio de la Protección Social, se tuvo en cuenta la información diagnóstica con base en la Clasificación Internacional 
de Enfermedades 10 ${ }^{\mathrm{a}}$ versión (CIE-10). donde TAB es definido como un desorden crónico y recurrente, caracterizado por fluctuaciones en el estado del ánimo y la energía, que afecta a más del 1\% del población mundial, siendo una de las principales causas de discapacidad en poblaciones jóvenes con compromiso que conlleva deterioro cognitivo y funcional.

Se tuvieron en cuenta los siguientes diagnósticos de TAB: episodio depresivo presente leve o moderado (F313), - TAB: episodio depresivo grave presente sin síntomas psicóticos (F314), TAB: episodio depresivo grave presente con síntomas psicóticos (F315), TAB: episodio mixto presente (F316). Asimismo, el número de pacientes atendidos y el número de consultas realizadas para los diagnósticos mencionados entre los años 2010 y 2015. Se llevaron a cabo 7111 atenciones (número de veces que los pacientes consultaron) que se distribuyeron para un total de 3252 pacientes (relación consulta paciente de $2: 1)$.

Se incluyeron variables numéricas y categóricas politómicas dentro del proceso. Se utilizó la plataforma Statistical Package for Social Sciences (SPSS $®$ ) versión 24 de la empresa IBM. Los resultados de $p(\alpha<0,05)$ fueron considerados como significativos. Se utilizó el estadígrafo $U$ de MannWhitney para las variables numéricas de distribución no normal. La prueba de Kolmogorov-Smirnov para medir el grado de concordancia existente entre la distribución del conjunto de datos y la distribución teórica específica.

\section{Consideraciones éticas}

En la investigación no se tuvo relación directa con los pacientes, los datos fueron aportados por la DTSC, en una base de datos con la respectiva custodia de la Universidad de Manizales; cada variable analizada no tenía identificación de los pacientes, por tanto, la confidencialidad de los datos, guardan los preceptos éticos y legales de la Resolución 8430 de 1993 del Ministerio de la Protección Social. El estudio contó con el aval del Comité de Ética de la Universidad de Manizales (Acta 005 de junio 2017).

\section{Resultados}

La prevalencia de TAB en el departamento de Caldas, en relación a la información recopilada para el 2010 fue en general de $0,03 \%$ y para mayores de 18 años fue de 0,08\%; para el 2015 en la población general, ascendió a $0,10 \%$ y para mayores de 18 años ascendió a 0,22\% (Tabla 1). La incidencia estuvo determinada por un incremento de 342 pacientes en 2010 a 969 pacientes en el 2015 (Tabla 2). También aumentó el número de atenciones por TAB en un $114 \%$ entre el 2010 y el 2015, se determinó un incremento porcentual promedio en la atención del $77,5 \%$ (Figuras 1 y 2 ).

La prueba de Kolmogorov-Smirnov con corrección de Lilliefors reveló que las variables numéricas recopiladas (Número de atenciones y de pacientes atendidos) presentaban una distribución diferente a la normal $(p=0,0001)$. Mediante el protocolo no paramétrico $U$ de Mann-Whitney, se encontró diferencia estadísticamente significativa entre el número de pacientes atendidos en relación a las categorías diagnósticas TAB-episodio depresivo leve o moderado y otros tipos de TAB (2450 frente a 1534 pacientes atendidos, $p<0,0001$ ).

Tabla 1. Prevalencia de TAB en el Departamento de Caldas, 2015 a 2017

\begin{tabular}{crrrr}
\hline Año & $\begin{array}{c}\text { Número de pacientes } \\
\text { atendidos (n) }\end{array}$ & $\begin{array}{c}\text { Prevalencia para el periodo } \\
\text { (población total) }\end{array}$ & $\begin{array}{c}\text { Prevalencia en > 18 años } \\
\text { (45\% de la población) }\end{array}$ & $\begin{array}{c}\text { Proyección Población } \\
\text { (DANE) 2010 - 2016 }\end{array}$ \\
\hline $\mathbf{2 0 1 0}$ & 342 & $0,03 \%$ & $0,08 \%$ & 978.362 \\
$\mathbf{2 0 1 1}$ & 186 & $0,02 \%$ & $0,04 \%$ & 980.281 \\
$\mathbf{2 0 1 2}$ & 419 & $0,04 \%$ & $0,09 \%$ & 982.202 \\
$\mathbf{2 0 1 3}$ & 545 & $0,06 \%$ & $0,12 \%$ & 984.128 \\
$\mathbf{2 0 1 4}$ & 791 & $0,08 \%$ & $0,18 \%$ & 986.061 \\
$\mathbf{2 0 1 5}$ & 969 & $0,10 \%$ & $0,22 \%$ & 988.003 \\
\hline
\end{tabular}

DANE: Departamento Nacional de Estadística 
Tabla 2. Casos nuevos atendidos con TAB e incidencia acumulada para mayores de 18 años

\begin{tabular}{crrrr}
\hline Año & $\begin{array}{c}\text { Número de pacientes } \\
\text { atendidos (n) }\end{array}$ & $\begin{array}{c}\text { Cambio porcentual por } \\
\text { periodo de atención }\end{array}$ & $\begin{array}{c}\text { Casos nuevos por año de } \\
\text { atención }\end{array}$ & $\begin{array}{c}\text { Incidencia acumulada } \\
>\mathbf{1 8} \text { años (45\% de la } \\
\text { población) }\end{array}$ \\
\hline $\mathbf{2 0 1 0}$ & 342 & 0 & 0 & 0 \\
$\mathbf{2 0 1 1}$ & 186 & $-45,6$ & -156 & $-0,0004$ \\
$\mathbf{2 0 1 2}$ & 419 & 22,5 & 233 & 0,0005 \\
$\mathbf{2 0 1 3}$ & 545 & 59,4 & 126 & 0,0003 \\
$\mathbf{2 0 1 4}$ & 791 & 131,3 & 246 & 0,0006 \\
$\mathbf{2 0 1 5}$ & 969 & 183,3 & 178 & 0,0004 \\
\hline
\end{tabular}

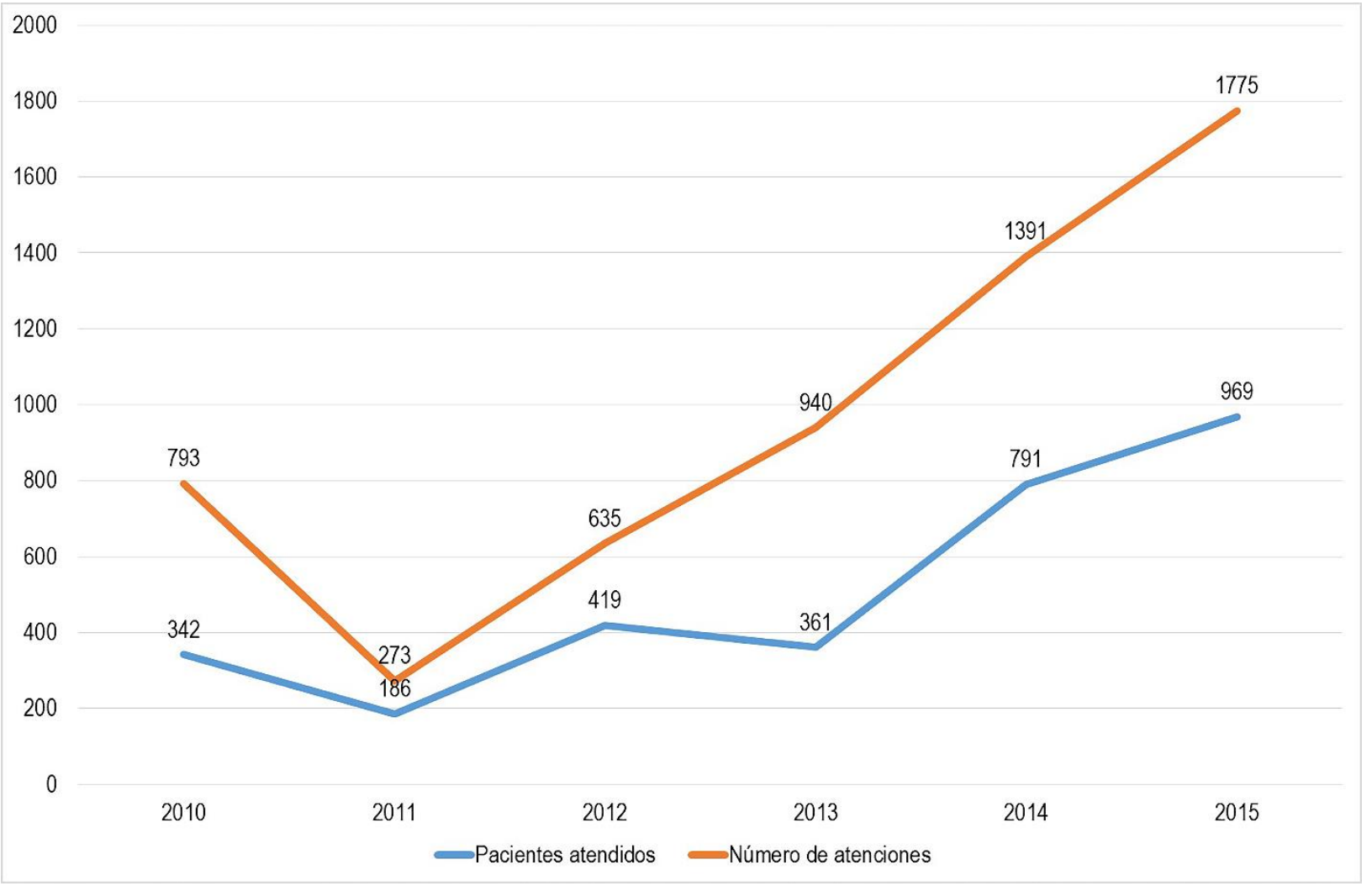

Figura 1. Pacientes atendidos y número de atenciones para TAB en Caldas, entre los años 2010 y 2015. 


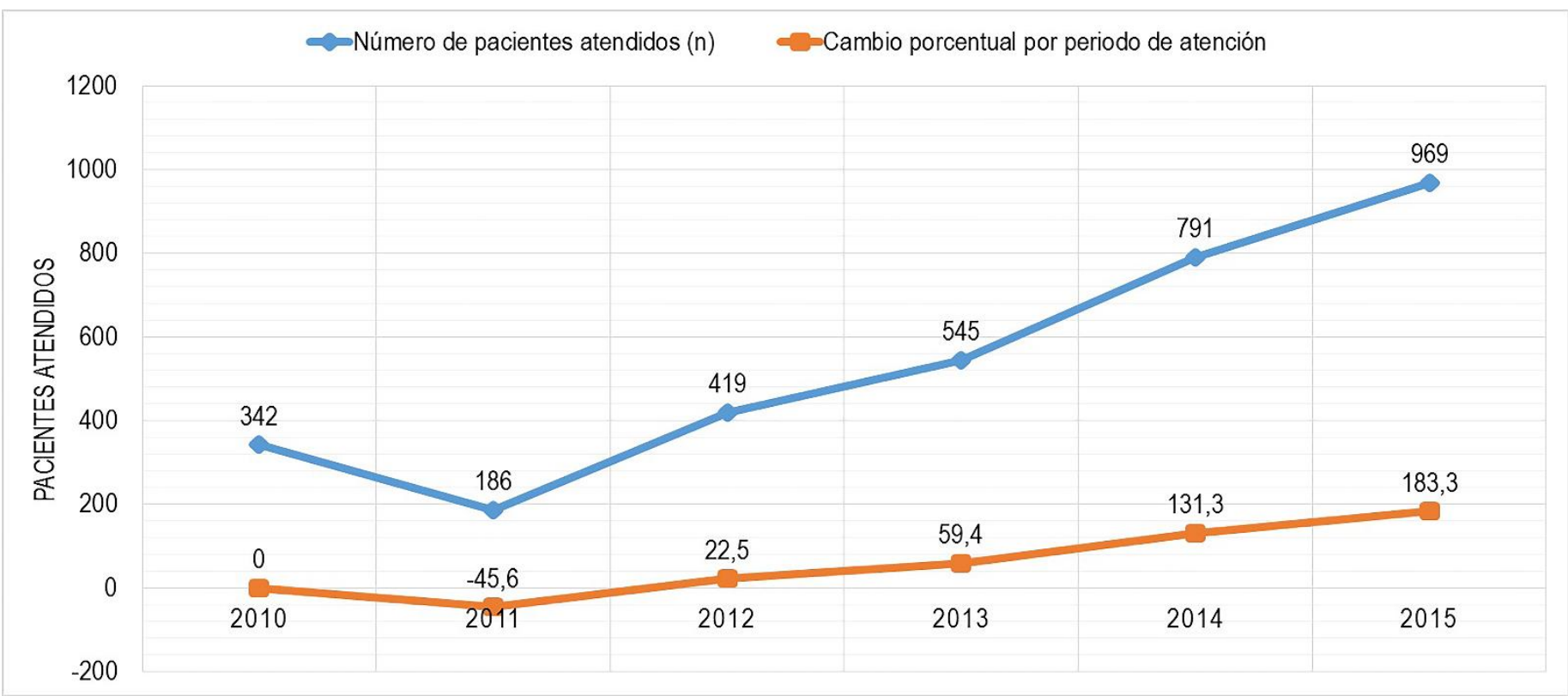

Figura 2. Evolución por casos atendidos y por cambio porcentual de los pacientes con diagnóstico de TAB para el periodo $2010-2015$.

\section{Discusión}

Se estima que entre $1 \%$ y $2 \%$ de la población mundial sufre TAB, según la Organización Mundial de la Salud (OMS), es la sexta causa de discapacidad en el mundo y la quinta en adultos, trayendo como consecuencia, una reducción de 9,2 años en la esperanza de vida de quienes la padecen; afecta las relaciones familiares, sociales y laborales, y se calcula que sólo un tercio de quienes la padecen buscan tratamiento(11,16). Para el diagnóstico del TAB, en el presente estudio se tuvo en cuenta la información diagnóstica con base en la CIE-10, correspondiente a la versión en español de la ICD (International Classification of Diseases) de la OMS, la cual clasifica y codifica las enfermedades, así como una amplia variedad de síntomas, signos, y otros factores relacionados con las estas(17).

La prevalencia conjunta de todos los trastornos bipolares ha sido calculada en los Estados Unidos en un 2,6\% de la población(18). Otros estudios muestran tasas de prevalencia más altas como el realizado en Zurich y en Hungría, con tasas de prevalencia del $5,5 \%$ y $5,1 \%$ respectivamente ${ }^{(19,20)}$. En el presente estudio, la prevalencia de TAB para el 2010 fue en general de $0,03 \%$ y para mayores de 18 años fue de 0,08\%; para el 2015 en la población general, ascendió a 0,10\% y para mayores de 18 años ascendió a 0,22\%, mostrando tasas similares a las encontradas por autores como: Luitel et al.,(21) $y$ Kudo et al.,(22) reportaron el 0,10\%; Moreno et al.,(23) el 0,11\%; Zhou et al.,(24) el 0,12\%; Leung et al.,(25) y Mirzaei et al.,(26) reportaron el 0,15\%; Merikangas et al.,(27) el 0,17\% y Charara et al.,(28) el 0,18\%, pero con un incremento a lo largo de los 5 años evaluados. Los estudios de cohorte o de seguimiento se utilizan para calcular la incidencia, pero son dispendiosos y toman largo tiempo para realizarlos, por ello, estos son escasos en salud mental. Un estudio reportó una tasa anual de incidencia de $0,5 \%$ para $\mathrm{TAB}^{(29)}$.

En este estudio se observó un incremento en la incidencia con un total de casos reportados de 342 en el año 2010 a 949 en el 2015. Algunos autores postulan que existe un incremento en la frecuencia del trastorno, debido al uso excesivo de antidepresivos, lo que aumenta el número de casos cíclicos, la utilización de sustancias estimulantes y bebidas con cafeína(27,29,30). Manifiestan que existen factores que incrementan su presentación y disminuyen la edad de inicio, tales como la luz artificial, la costumbre de acostarse tarde o dormir menos horas, y llevar un estilo de vida más intenso con gran cantidad de estímulos ambientales(30).

El riesgo de morbilidad a lo largo de la vida para el trastorno bipolar es del 0,8\%, y ha aumentado la 
frecuencia del padecimiento, mediante un fenómeno denominado "tendencia secular"(31), es decir, algo que lejos de ser cíclico o pasajero llega para quedarse por un largo período de tiempo. Estas tendencias generan cambios importantes en las sociedades y afectan el comportamiento de las personas, desencadenado muchas veces por factores psicosociales adversos, familias disfuncionales, y problemas económicos. Puede definirse como cambio secular dentro de los procesos microadaptativos que generan variación en las poblaciones humanas, a las modificaciones somáticas y fisiológicas que son resultado de las variaciones ambientales(32).

La evolución secular entonces, se ve afectada por las condiciones ambientales de la población, y estos cambios se aceptan como positivos y negativos. Por otro lado, debe tenerse en cuenta, además, los factores epigenéticos, que hacen referencia a las interacciones entre genes y ambiente, que se producen en los organismos, y el conjunto de reacciones químicas y demás procesos que modifican la actividad del ADN (Ácido Desoxiribonucleico) sin alterar su secuencia. Tiene en cuenta cambios de larga data en la expresión genética, que son regulados mediante mecanismos transcripcionales,

posttranscripcionales, translacionales y/o post-translacionales, tales como la metilación del ADN, la hidroximetilación del ADN, modificaciones en las histonas y ARNs (Ácidos Ribonucleicos) que no codifican, los cuales no modifican la estructura del $\operatorname{ADN}(33)$. Asimismo, algunos factores como el aumento significativo en el consumo de drogas de abuso como el alcohol, pueden comportarse como factores epigenéticos, que podría ser responsable del incremento en la presentación del TAB.

Es importante considerar que los abordajes diagnósticos pueden estar influenciados por variables como diferencias culturales al experimentar los síntomas, estigma para hablar acerca de los problemas psicológicos, prevalencia de factores de riesgo y acceso al cuidado de la salud mental(34), y que los métodos que se utilizan para hacer el diagnóstico, han cambiado a través del tiempo; tal es el caso de las entrevistas diagnósticas estandarizadas, las cuales son rara vez usadas por los clínicos, y se correlacionan pobremente al compararlas con las evaluaciones clínicas(35); algo confirmado en otros estudios(36), donde evaluaron la sensibilidad y especificidad entre la entrevista diagnóstica internacional compuesta versión 3.0 (world mental health, CIDI) con la evaluación clínica estandarizada versión I (SCID-I), y encontraron moderados niveles de sensibilidad y especificidad para el diagnóstico del TAB.

Los abordajes diagnósticos también pueden cambiar de acuerdo a las variaciones en escalas de medición como el DSM (Diagnostic and Statistical Manual of Mental Disorders)(37), y aunque este ha sido aceptado universalmente como la última autoridad en psicopatología y diagnosis, sus criterios diagnósticos incluyen solamente algunos síntomas característicos. Así mismo, el valor pronóstico (duración, curso y severidad de la enfermedad), fueron definidos arbitrariamente y convencionalmente, mostrando por lo tanto poco acuerdo entre los sistemas de medición DSM y el ICD(38,39).

El uso de medicamentos hace parte de la realidad social, como una posibilidad para conseguir alivio o solución a los problemas de salud de las poblaciones. La prescripción hace parte fundamental del acto médico, para lo cual, es imprescindible conocer las indicaciones específicas de los medicamentos, y hacerlo basado en un diagnóstico, lo más preciso posible ${ }^{(40)}$. Dentro de los procesos de cubrimiento de medicamentos del Plan Obligatorio de Salud (POS), existen restricciones específicas para medicamentos, que tienen que ver con su uso en la práctica clínica, sin indicaciones aprobadas para una determinada patología, dosis o población(41).

Subsisten límites legales dentro de los procesos de autorización para el suministro de medicamentos, que generan barreras para la prescripción. A partir del año 2013 con la ampliación del POS mediante la Resolución 5521, se mejoró el espacio para la formulación de mayor cantidad de antipsicóticos atípicos, lo que facilitó el acceso para una mayor población a los mismos(41). Sin embargo, puede observarse, que para que los pacientes con otras patologías mentales, tengan fácil acceso a la formulación incluida en el POS y se beneficien del uso de estos medicamentos, los médicos pueden incluirlos dentro del grupo de pacientes diagnosticados con $\mathrm{TAB}$, en principio de beneficencia. Esta práctica denominada "off label", hace referencia al uso fuera de la indicación, o para una indicación no habitual, una indicación no oficial o no autorizada, o de uso alternativo o uso 
desvirtuado, es habitual, y hace referencia a la formulación de medicamentos en situaciones no contempladas dentro de su ficha técnica, o para usos no aprobados por los entes reguladores. Esta práctica, alcanza hasta un $11 \%$ de la totalidad de la prescripción en Estados Unidos, un 21\% en Canadá y un $40 \%$ en Australia( ${ }^{(42)}$.

\section{Conclusiones}

Se observa un marcado incremento del diagnóstico de TAB en Caldas, posiblemente debido a problemas socioculturales, causas epigenéticas o problemas de formulación tipo off-label. Estos hallazgos revisten importancia, toda vez que llama la atención a los clínicos, con relación a la responsabilidad a la hora de establecer un diagnóstico preciso, mostrando que es indispensable, evitar utilizar otros diagnósticos en beneficio de pacientes con otras patologías, a la vez que marcan el camino, en la investigación epidemiológica de las enfermedades mentales, no solamente en el departamento, sino en todo el territorio nacional.

Los resultados en la investigación posiblemente se deben a fenómenos de tendencia secular, que afectan el comportamiento de las personas, desencadenado muchas veces por factores psicosociales adversos, familias disfuncionales, y problemas económicos. Algunos factores como el aumento significativo en el consumo de drogas de abuso como el alcohol, que pueden comportarse como factores epigenéticos, o el diagnóstico de $\mathrm{TAB}$ en pacientes que requieren acceder a medicamentos no permitidos en el POS para otras patologías.

Se sugiere revisar en otros departamentos si existe incremento en la incidencia y prevalencia, y los factores asociados, para conocer más a fondo el mapa epidemiológico del país, en lo relacionado a los trastornos mentales.

Conflicto de intereses: Los autores declaran no tener ningún tipo de conflicto de interés.

\section{Referencias}

1. Li H, Mukherjee D, Krishnamurthy VB, Millett C, Ryan KA, Zhang $\mathrm{L}$, et al. Use of ecological momentary assessment to detect variability in mood, sleep and stress in bipolar disorder. BMC Res Notes. 2019;12(1):791. doi: 10.1186/s13104-019-4834-7.

2. McCormick U, Murray B, McNew B. Diagnosis and treatment of patients with bipolar disorder: A review for advanced practice nurses. J Am Assoc Nurse Pract. 2015;27(9):53042. doi: $10.1002 / 2327-6924.12275$

3. Bruschi A, Mazza M, Camardese G, Calò S, Palumbo C, Mandelli L, et al. Psychopathological Features of Bipolar Depression: Italian Validation of the Bipolar Depression Rating Scale (I-BDRS). Front Psychol. 2018;9:1047. doi: 10.3389/fpsyg.2018.01047

4. Patel RS, Virani S, Saeed H, Nimmagadda S, Talukdar J, Youssef NA. Gender Differences and Comorbidities in U.S. Adults with Bipolar Disorder. Brain Sci. 2018;1;8(9):168. doi: $10.3390 /$ brainsci8090168

5. Cerimele J, Fortney J, Pyne J, Curran G. Bipolar disorder in primary care: a qualitative study of clinician and patient experiences with diagnosis and treatment. Fam Pract. 2019;36(1): 32-37. doi: 10.1093/fampra/cmy019

6. Kim Y, Santos R, Gage FH, Marchetto MC. Molecular Mechanisms of Bipolar Disorder: Progress Made and Future Challenges. Front Cell Neurosci. 2017;11:30. doi: 10.3389/fncel.2017.00030

7. Leung CM, Yim CL, Yan CT, Chan CC, Xiang YT, Mak AD, et al. The Bipolar II Depression Questionnaire: A Self-Report Tool for Detecting Bipolar II Depression. PLoS One. 2016;11(3):e0149752. doi: 10.1371/journal.pone.0149752

8. Neil ER, Winkelmann ZK, Edler JR. Defining the Term "Overuse": An Evidence-Based Review of Sport Epidemiology Literature. J Athl Train. 2018;53(3):279-281. doi: 10.4085/1062-6050-84-16

9. Fajardo-Gutiérrez A. Medición en epidemiología: prevalencia, incidencia, riesgo, medidas de impacto. Rev. alerg. Méx. 2017;64(1):109-120. doi: 10.29262/ram.v64i1.252

10. Scotti L, Franchi M, Marchesoni A, Corrao G. Prevalence and incidence of psoriatic arthritis: A systematic review and meta-analysis. Semin Arthritis Rheum. 2018;S00490172(17):30387-6. doi.org/10.1016/j.semarthrit.2018.01.003

11. Bauer AM, Hodsdon S, Bechtel JM, Fortney JC. Applying the Principles for Digital Development: Case Study of a Smartphone App to Support Collaborative Care for Rural Patients With Posttraumatic Stress Disorder or Bipolar Disorder. J Med Internet Res. 2018;20(6):e10048. doi: 10.2196/10048

12. Clements C, Kapur N, Jones SH, Morriss R, Peters S Qualitative investigation of relatives' and service users' experience of mental healthcare for suicidal behaviour in bipolar disorder. BMJ Open. 2019;9(11):e030335. doi: 10.1136/bmjopen-2019-030335

13. O'Rourke N, Heisel MJ, Canham SL, Sixsmith A. Predictors of suicide ideation among older adults with bipolar disorder. PLoS One. 2017;12(11):e0187632. doi: 10.1371/journal.pone.0187632

14. Dome P, Rihmer Z, Gonda X. Suicide Risk in Bipolar Disorder: A Brief Review. Medicina (Kaunas). 2019;55(8):403. doi: 10.3390/medicina55080403

15. De Oliveira L, Portugal LCL, Pereira M, Chase HW, Bertocci M, Stiffler R, et al. Predicting Bipolar Disorder Risk Factors in Distressed Young Adults From Patterns of Brain Activation to Reward: A Machine Learning Approach. Biol Psychiatry Cogn Neurosci Neuroimaging. 2019;4(8):726733. doi: 10.1016/j.bpsc.2019.04.005

16. Organización Mundial De La Salud (OMS). Guía de intervención mhGPAP (versión 2.0): para los trastornos mentales, neurológicos y por uso de sustancias psicoactivas 
en nivel no especializado. Ginebra: OMS; 2016. 1. [Internet]. [cited 2019 Mar 5]. Available from: https://www.paho.org/mhgap/es/doc/GuiamhGAP.pdf?ua $=1$

17. World Health Organization. The ICD-10 classification of mental and behavioural disorders: diagnostic criteria for research; 1993. [Internet]. [citado 10 Feb 2019]. Disponible en: World Health Organization. https://scholar.google.com/scholar?q=The+ICD$10+$ Classification+of+Mental+and+Behavioural+Disorders:+ Diagnostic+Criteria+for+Research+World+Health+Organisa tion+Geneva+WHO+1993+http://www.who.int/classificati ons/icd/en/GRNBOOK.pdf+

18. Duffy A, Vandeleur C, Heffer N, Preisig M. The clinical trajectory of emerging bipolar disorder among the high-risk offspring of bipolar parents: current understanding and future considerations. Int J Bipolar Disord. 2017;5(1):37. doi: 10.1186/s40345-017-0106-4

19. Hunsberger JG, Chibane FL, Elkahloun AG, Henderson R, Singh R, Lawson J, et al. Novel integrative genomic tool for interrogating lithium response in bipolar disorder. Transl Psychiatry. 2015;5(2):e504. doi: 10.1038/tp.2014.139

20. Spoorthy MS, Chakrabarti S, Grover S. Comorbidity of bipolar and anxiety disorders: An overview of trends in research. World J Psychiatry. 2019;9(1):7-29. doi: 10.5498/wjp.v9.i1.7

21. Luitel NP, Garman EC, Jordans MJD, Lund C. Change in treatment coverage and barriers to mental health care among adults with depression and alcohol use disorder: a repeat cross sectional community survey in Nepal. BMC Public Health. 2019;19(1):1350. doi: 10.1186/s12889-0197663-7

22. Kudo S, Tomita T, Sugawara N, Sato Y, Ishioka M, Tsuruga K, et al. The low level of understanding of depression among patients treated with antidepressants: a survey of 424 outpatients in Japan. Neuropsychiatr Dis Treat. 2015;11:2811-6. doi: 10.2147/NDT.S93657

23. Moreno-Küstner B, Martín C, Pastor L. Prevalence of psychotic disorders and its association with methodological issues. A systematic review and meta-analyses. PLoS One. 2018;13(4):e0195687. doi: 10.1371/journal.pone.0195687

24. Zhou X, Bi B, Zheng L, Li Z, Yang H, Song H, et al. The prevalence and risk factors for depression symptoms in a rural Chinese sample population. PLoS One. 2014;9(6):e99692. doi: 10.1371/journal.pone.0099692

25. Leung CM, Yim CL, Yan CT, Chan CC, Xiang YT, Mak AD, et al. The Bipolar II Depression Questionnaire: A Self-Report Tool for Detecting Bipolar II Depression. PLoS One. 2016;11(3):e0149752. doi: 10.1371/journal.pone.0149752

26. Mirzaei M, Yasini Ardekani SM, Mirzaei M, Dehghani A. Prevalence of Depression, Anxiety and Stress among Adult Population: Results of Yazd Health Study. Iran J Psychiatry. 2019;14(2):137-146. [Internet]. [cited 2019 Aug 9]. Available /pmc/articles/PMC6702282/?report=abstract

27. Merikangas KR, Jin R, He J-P, Kessler RC, Lee S, Sampson NA, et al. Prevalence and Correlates of Bipolar Spectrum Disorder in the World Mental Health Survey Initiative. Arch Gen Psychiatry. 2011;68(3):241-51. doi: 10.1001/archgenpsychiatry.2011.12

28. Charara R, Forouzanfar $M$, Naghavi M, Moradi-Lakeh $M$, Afshin A, Vos T, et al. The Burden of Mental Disorders in the
Eastern Mediterranean Region, 1990-2013. PLoS One. 2017;12(1):e0169575. doi: 10.1371/journal.pone.0169575

29. Roos KG, Marshall SW. Definition and usage of the term "overuse injury" in the US high school and collegiate sport epidemiology literature: a systematic review. Sports Med. 2014;44(3):405-421. doi:10.1007/s40279-013-0124-z

30. Dolsen MR, Asarnow LD, Harvey AG. Insomnia as a transdiagnostic process in psychiatric disorders. Curr Psychiatry Rep. 2014;16(9):471. doi: 10.1007/s11920-0140471-y

31. Montesinos JE. Bipolaridad. Presentación familiar. Genética del trastorno. Alcmeon. 2014;19(1):43-51. [Internet]. [citado 12 enero 2019]. Disponible en: https://www.alcmeon.com.ar/19/06_bipolaridad_montesin os.pdf

32. Van Wieringen JC. Secular growth changes. En Human Growth. A comprehensive treatise, 2nd ed. Vol 3: Methodology; Ecological, Genetic and Nutritional Effects of Growth, editores F. Falkner, J.M. Tanner. New York: Plenum Press;1986.

33. Berdasco M, Manel Esteller M. Clinical epigenetics: seizing opportunities for translation. Nature Reviews Genetics. 2019;20:109-127. doi: 10.1038/s41576-018-0074-2

34. Moreira ALR, Van Meter A, Genzlinger J, Youngstrom EA. Review and Meta-Analysis of Epidemiologic Studies of Adult Bipolar Disorder. J Clin Psychiatry. 2017;78(9):e1259e1269. [Internet]. [cited 2019 may 9]. Available from: https://reference.medscape.com/medline/abstract/29188 905

35. Scott MJ. Improving Access to Psychological Therapies (IAPT) - The Need for Radical Reform. J Health Psychol. 2018;23(9):1136-1147. doi: 10.1177/1359105318755264

36. Montoya-González LE, Restrepo-Bernal DP, Mejía-Montoya R, Bareno-Silva J, Sierra-Hincapié G, Torres de Galvis Y, et al. Sensibilidad y especificidad entre la Entrevista Diagnóstica Internacional Compuesta versión 3.0 (World Mental Health, CIDI) con la Evaluación Clínica Estandarizada versión I (SCID-I) en la Encuesta de Salud Mental de la ciudad de Medellín 2012. Rev colomb psiquiat. 2016;45(1):22-27. doi: 10.1016/j.rcp.2015.07.001

37. Faravelli C, Castellini G, Landi M, Brugnera A. Are Psychiatric Diagnoses an Obstacle for Research and Practice? Reliability, Validity and the Problem of Psychiatric Diagnoses. The Case of GAD. Clin Pract Epidemiol Ment Health. 2012;8:12-5. doi: 10.2174/1745017901208010012

38. Keromnes G, Motillon T, Coulon N, Berthoz A, Du Boisgueheneuc F, Wehrmann M, et al. Self-other recognition impairments in individuals with schizophrenia: a new experimental paradigm using a double mirror. NPJ Schizophr. 2018;4(1):24. doi: 10.1038/s41537-018-0065-5

39. Maibing $\mathrm{CF}$, Pedersen $\mathrm{CB}$, Benros ME, Mortensen PB, Dalsgaard S, Nordentoft M. Risk of Schizophrenia Increases After All Child and Adolescent Psychiatric Disorders: A Nationwide Study. Schizophr Bull. 2015;41(4):963-70. doi: 10.1093/schbul/sbu119

40. Organización Médica Colegial [Internet]. Madrid: The Association; [actualizado 20 de enero 2008; citado 12 enero 2019]. [aprox. 2 pantallas]. Disponible en: https://www.cgcom.es/sites/default/files/alegaciones_pre scripcion.pdf

41. De Souza Gonçalves A, Moreira A, Gusmão A, Ferrarez M. Use of unlicensed and off-label drugs in neonates in a Brazilian university hospital. Braz. J. Pharm. Sci. 
2017;53(3):e00252.

doi:

$10.1590 / s 2175-$ 97902017000300252

42. López J. Papel de la Farmacovigilancia en el uso off label de medicamentos [Internet]. Colombia: CIMUN, Universidad Nacional de Colombia; 2014. [citado 9 Ago 2019].
Disponible

en:

http://www.invima.gov.co/documents/20143/844940/1-

11-Seguridad-y-vigilancia-en-el-uso-off-label.pdf/f75b59c0efa1-53df-19f4-00c61ffc323c 